\title{
A variational inequality method for computing a normalized equilibrium in the generalized Nash game
}

\author{
Jian $\mathrm{Hou}^{1^{*}}$, Zong-Chuan Wen ${ }^{2}$ and Zhu Wen ${ }^{2}$
}

* Correspondence:
houjiantougao@163.com
${ }^{1}$ Institute of ORCT, School of
Mathematical Sciences, Dalian
University of Technology, Dalian
116024, China
Full list of author information is
available at the end of the article

* Correspondence: houjiantougao@163.com Mathematical Sciences, Dalian University of Technology, Dalian Full list of author information is available at the end of the article

\begin{abstract}
The generalized Nash equilibrium problem is a generalization of the standard Nash equilibrium problem, in which both the utility function and the strategy space of each player may depend on the strategies chosen by all other players. This problem has been used to model various problems in applications but convergent solution algorithms are extremely scare in the literature. In this article, we show that a generalized Nash equilibrium can be calculated by solving a variational inequality (VI). Moreover, conditions for the local superlinear convergence of a semismooth Newton method being applied to the VI are also given. Some numerical results are presented to illustrate the performance of the method.
\end{abstract}

Keywords: Nash equilibrium problem, generalized Nash equilibrium problem, variational inequality, semismooth function, superlinear convergence

\section{Introduction}

In this article, We consider the generalized Nash equilibrium problem (GNEP). To this end, we first recall the definition of the Nash equilibrium problem (NEP). There are $N$ players, each player $v \in\{1, \ldots, N\}$ controls the variables $x^{v} \in \Re^{n_{\nu}}$. All players' strategies are collectively denoted by a vector $x=\left(x^{1}, \ldots, x^{N}\right)^{T} \in \mathfrak{R}^{n}$, where $n=n_{1}+\ldots+n_{N}$. To emphasize the $v$ th player's variables within the vector $x$, we sometimes write $x=\left(x^{v}, x^{-v}\right)^{T}$, where $x^{-v} \in \Re^{n_{-v}}$ subsumes all the other players' variables.

Let $\theta^{v}: \Re^{n} \rightarrow \Re$ be the $v$ th player's payoff (or loss or utility) function, and let $X^{v} \subseteq \Re^{n_{v}}$ be the strategy set of player $v$. Then, $x^{*}=\left(x^{*, 1}, \ldots, x^{*, N}\right)^{T} \in \Re^{n}$ is called a Nash equilibrium, or a solution of the NEP, if each block component $x^{* \prime, v}$ is a solution of the optimization problem

$$
\begin{array}{ll}
\min _{x^{\nu}} & \theta^{\nu}\left(x^{\nu}, x^{*,-\nu}\right) \\
\text { s.t. } & x^{\nu} \in X^{\nu} .
\end{array}
$$

On the other hand, in a GNEP, each player's strategy belongs to a set $X_{v}\left(x^{-v}\right) \subseteq \Re^{n_{v}}$ that depends on the rival players' strategies. The aim of each player $v$, given the other players' strategies $x^{-v}$, is to choose a strategy $x^{v}$ that solves the minimization problem

(c) 2012 Hou et al; licensee Springer. This is an Open Access article distributed under the terms of the Creative Commons Attribution License (http://creativecommons.org/licenses/by/2.0), which permits unrestricted use, distribution, and reproduction in any medium, provided the original work is properly cited. 


$$
\begin{aligned}
\min _{x^{\nu}} & \theta^{v}\left(x^{v}, x^{-v}\right) \\
\text { s.t. } & x^{v} \in X_{v}\left(x^{-v}\right) .
\end{aligned}
$$

The GNEP is the problem of finding a vector $x^{*}$ such that each player's strategy $x^{*}, v$ satisfies

$$
\theta^{v}\left(x^{*, v}, x^{*,-v}\right) \leq \theta^{v}\left(y^{v}, x^{*,-v}\right), \quad \forall y^{v} \in X_{v}\left(x^{*,-v}\right) .
$$

Such a vector $x^{*}$ is called a generalized Nash equilibrium or, more simply a solution of the GNEP.

In this article, we focus on a special class of GNEPs referred to as jointly convex GNEPs. More precisely, we assume that there is a closed and convex set $X \subseteq \Re^{n}$, which represents the joint constraints of all the players, such that

$$
X_{v}\left(x^{-v}\right):=\left\{x^{\nu} \in \Re^{n_{\nu}} \mid\left(x^{\nu}, x^{-v}\right) \in X\right\},
$$

for all $v=1, \ldots, N$. This condition results to be verified in several applications. Throughout this article, we assume that the set $X$ can be represented as

$$
X=\left\{x \in \mathfrak{R}^{n} \mid g(x) \leq 0\right\}
$$

for some function $g: \Re^{n} \rightarrow \Re^{m}$. Additional equality constraints are also allowed, but for notational simplicity, we prefer not to include them explicitly. In many cases, a player $v$ might have some additional constraints depending on his decision variables only. However, these additional constraints can be viewed as part of the joint constraints $g(x) \leq 0$, so, we include these latter constraints in the former ones.

Throughout this article, we make the following blanket assumptions.

Assumption 1.1 (i) The utility functions $\theta^{v}$ are twice continuously differentiable and as a function of $x^{v}$ along, convex.

(ii) The function $g$ is twice continuously differentiable, its components $g_{i}$ are convex (in $x$ ), and the corresponding strategy space $X$ defined by (1.2) is nonempty.

The convexity assumptions are standard in the context of GNEPs. The smoothness assumptions are also very natural since our aim is to develop locally fast convergent methods for the solution of GNEPs.

The GNEP was formally introduced by Debreu [1] as early as 1952, but it is only from the mid-1990s that the GNEP attracted much attention because of its capability of modeling a number of interesting problems in economy computer science, telecommunications, and deregulated markets (e.g., see [2-4]). Another approach for solving the GNEP is based on the Nikaido-Isoda function. Relaxation methods and proximallike methods using the Nikaido-Isoda function are investigated in [5-7]. A regularized version of the Nikaido-Isoda function was first introduced in [8] for standard NEPs then further investigated by Heusinger and Kanzow [9], they reformulated the GNEP as a constrained optimization problem with continuously differentiable objective function.

Motivated by the fact that a standard NEP can be reformulated as a variational inequality problem (VI for short), see, for example, [10,11], Harker [12] characterized the GNEP as a quasi-variational inequality(QVI). But unlike VI, there are few efficient methods for solving QVI, and therefore such a reformulation is not used widely in designing implementable algorithms. On the other hand, it was noted in [13], for 
example, that certain solutions of the GNEP (the normalized Nash equilibria, to be defined later) can be found by solving a suitable standard VI associated to the GNEP.

Here, we further investigate the properties of the normalized Nash equilibria. The rest of the article is organized as follows. Section 2 gives some preliminaries. In Section 3 , we use the fact that the normalized Nash equilibria can be found by solving a suitable VI, we reformulate the VI associated to the GNEP as a semismooth system of equations and the nonsingularity of the B-subdifferential for the system is explored. Finally, in Section 4, we implement a semismooth Newton method to some examples of the GNEP.

We use the following notations throughout the article. A function $G: \Re^{n} \rightarrow \Re^{t}$ is called a $C^{k}$-function if it is $k$ times continuously differentiable. For a differentiable function $g: \Re^{n} \rightarrow \Re^{m}$, the Jacobian of $g$ at $x \in \mathfrak{R}^{n}$ is denoted by $\mathcal{J} g(x)$, and its transposed by $\nabla g(x)$. Given a differentiable function $\Psi: \Re^{n} \rightarrow \Re$, the symbol $\nabla_{x^{\nu}} \Psi(x)$ denotes the partial gradient with respect to $x^{v}$-part only, and $\nabla_{x^{\nu} x^{\mu}}^{2} \Psi(x)$ denotes the second-order partial derivative with respect to $x^{\nu}$-part and $x^{\mu}$-part. For a function $f: \Re^{n} \times \Re^{n} \rightarrow \Re, \quad f(x, \cdot): \Re^{n} \rightarrow \Re$ denotes the function with $x$ being fixed. For vectors $x, y \in \Re^{n},\langle x, y\rangle$ denotes the inner product defined by $\langle x, y\rangle:=x^{T} y$ and $x \perp y$ means $\langle x, y\rangle=0$.

\section{Preliminaries}

Let $F: \Re^{n} \rightarrow \Re^{m}$ be a locally Lipschitz continuous function. By Rademacher's theorem, $F$ is differentiable almost everywhere. Let $D_{F}$ denote the set of points where $F$ is differentiable. Then, the Bouligand-subdifferential of $F$ at $x$ is given by (see [14]),

$$
\partial_{B} F(x):=\left\{H \in \Re^{m \times n} \mid \exists\left\{x^{k}\right\} \subseteq D_{F}: x^{k} \rightarrow x, H=\lim _{k \rightarrow \infty} \mathcal{J} F\left(x^{k}\right)\right\} .
$$

Its convex hull

$$
\partial F(x):=\operatorname{conv}\left\{\partial_{B} F(x)\right\}
$$

is Clarke's generalized Jacobian of $F$ at $x$ (see [15]).

Based on this notation, we next recall the definition of a semismooth function. This concept was firstly introduced by Mifflin [16] for real-valued mappings and extended by Qi and Sun [17] to vector-valued mappings.

Definition 2.1 Let $\Phi: \mathcal{O} \subseteq \Re^{n} \rightarrow \Re^{m}$ be a locally Lipschitz continuous function on the open set $\mathcal{O}$. We say that $\Phi$ is semismooth at a point $x \in \mathcal{O}$ if

(i) $\Phi$ is directionally differentiable at $x$; and

(ii) for any $\Delta x \in X$ and $V \in \partial \Phi(x+\Delta x)$ with $\Delta x \rightarrow 0$,

$$
\Phi(x+\Delta x)-\Phi(x)-V(\Delta x)=\mathrm{o}(\|\Delta x\|) .
$$

Furthermore, $\Phi$ is said to be strongly semismooth at $x \in \mathcal{O}$ if $\Phi$ is semismooth at $x$ and for any $\Delta x \in X$ and $V \in \partial \Phi(x+\Delta x)$ with $\Delta x \rightarrow 0$,

$$
\Phi(x+\Delta x)-\Phi(x)-V(\Delta x)=\mathrm{O}\left(\|\Delta x\|^{2}\right) .
$$

In the study of algorithms for locally Lipschitzian systems of equations, the following regularity condition plays a role similar to that of the nonsingularity of the Jacobian in the study of algorithms for smooth systems of equations. 
Definition 2.2 Let $G: \Re^{n} \rightarrow \Re^{n}$ be Lipschitzian around $x, G$ is said to be BD-regular at $x$ if all the elements in $\partial_{B} G(x)$ are nonsingular. If $\bar{x}$ is a solution of the system $G(x)=$ 0 and $G$ is BD-regular at $\bar{x}$, then $\bar{x}$ is called a BD-regular solution of this system.

Given a closed convex set $K \subseteq \Re^{n}$ and a continuous function $G: K \rightarrow \Re^{n}$, solving the VI defined by $K$ and $G$ (which is denoted by $\operatorname{VI}(G, K)$ ) means finding a vector $x \in K$ such that

$$
G(x)^{T}(y-x) \geq 0, \quad \text { for all } y \in K .
$$

Define the function $F: \Re^{n} \rightarrow \Re^{n}$ by

$$
F(x):=\left(\begin{array}{c}
\nabla_{x^{1}} \theta^{1}(x) \\
\vdots \\
\nabla_{x^{N}} \theta^{N}(x)
\end{array}\right),
$$

we state a result due to [13] which will be used later.

Lemma 2.1 Suppose that the GNEP satisfies Assumption 1.1 and assume further that the sets $X_{v}\left(x^{-v}\right)$ are defined by (1.1) with $X$ closed and convex. Then, every solution of the $\operatorname{VI}(F, X)$ is a solution of the GNEP.

\section{The nonsmooth equation reformulation and nonsingularity conditions}

Consider the GNEP from Section 1 with utility functions $\theta^{v}$ and a strategy set $X$ satisfying the requirements from Assumption 1.1. In this section, our aim is to show that the GNEP can be reformulated as a nonsmooth equation and then we present several conditions guaranteeing the BD-regularity condition of the equation.

Suppose that $x$ is a solution of the GNEP. Then if for player $v$, a suitable constraint qualification (like the slater condition) holds, it follows that there exists a Lagrange multiplier $\lambda^{v} \in \Re^{m}$ such that the Karush-Kuhn-Tucker (KKT) conditions

$$
\begin{gathered}
\nabla_{x^{\nu}} \theta^{v}\left(x^{\nu}, x^{-v}\right)+\nabla_{x^{\nu}} g\left(x^{\nu}, x^{-v}\right) \lambda^{v}=0, \\
0 \leq \lambda^{\nu} \perp-g\left(x^{\nu}, x^{-v}\right) \geq 0
\end{gathered}
$$

are satisfied.

Let us consider the KKT conditions for the $\mathrm{VI}(F, X)$. Assuming that a suitable constraint qualification holds at a solution $x$, the KKT conditions can be expressed as

$$
\begin{aligned}
& F(x)+\nabla g(x) \lambda=0, \\
& 0 \leq \lambda \perp-g(x) \geq 0,
\end{aligned}
$$

which is equivalent to

$$
\begin{gathered}
\left(\begin{array}{c}
\nabla_{x^{1}} \theta^{1}(x) \\
\vdots \\
\nabla_{x^{N}} \theta^{N}(x)
\end{array}\right)+\left(\begin{array}{c}
\nabla_{x^{1}} g(x) \\
\vdots \\
\nabla_{x^{N}} g(x)
\end{array}\right) \lambda=0, \\
0 \leq \lambda \perp-g(x) \geq 0 .
\end{gathered}
$$

The next lemma from [13] relates the normalized Nash equilibria to the KKT conditions (3.3).

Lemma 3.1 (i) Let $x$ be a solution of VI(F,X) at which the KKT conditions (3.3) hold. Then $x$ is a solution of the GNEP (normalized Nash equilibria) at which the KKT conditions (3.1) hold with $\lambda^{1}=\lambda^{2}=\ldots=\lambda^{N}=\lambda$. 
(ii) Viceversa, let $x$ be a solution of the GNEP at which KKT conditions (3.1) hold with $\lambda^{1}=\lambda^{2}=\ldots=\lambda^{N}$. Then $x$ is a solution of $V I(F, X)$.

Using the minimum function $\varphi: \Re \times \Re \rightarrow \Re, \varphi(a, b):=\min \{a, b\}$, the KKT conditions (3.2) can equivalently be written as the nonlinear system of equations

$$
\Phi(\omega):=\Phi(x, \lambda)=0,
$$

where $\Phi: \mathfrak{R}^{n+m} \rightarrow \mathfrak{R}^{n+m}$ is defined by

$$
\Phi(\omega)=\Phi(x, \lambda):=\left(\begin{array}{c}
L(x, \lambda) \\
\phi(-g(x), \lambda)
\end{array}\right),
$$

and

$$
\begin{aligned}
& L(x, \lambda):=F(x)+\nabla g(x) \lambda, \\
& \phi(-g(x), \lambda):=\left(\varphi\left(-g_{1}(x), \lambda_{1}\right), \ldots, \varphi\left(-g_{m}(x), \lambda_{m}\right)\right)^{T} \in \Re^{m} .
\end{aligned}
$$

From Assumption 1.1, we know that $\Phi$ is semismooth.

In the following, our aim is to present several conditions guaranteeing that all elements in the generalized Jacobian $\partial \Phi(\omega)$ (and hence in the B-subdifferential $\partial_{B} \Phi(\omega)$ ) are nonsingular. Our first result gives a description of the structure of the matrices in the generalized Jacobian $\partial \Phi(\omega)$.

Lemma 3.2 Let $\omega=(x, \lambda) \in \mathfrak{R}^{n+m}$. Then, each element $H \in \partial \Phi(\omega)^{T}$ can be represented as follows:

$$
H=\left[\begin{array}{cc}
\nabla_{x} L(\omega)-\nabla g(x) D_{a}(\omega) \\
\nabla g(x)^{T} & D_{b}(\omega)
\end{array}\right]
$$

where $D_{a}(\omega):=\operatorname{diag}\left(a_{1}(\omega), \ldots, a_{m}(\omega)\right), D_{b}(\omega):=\operatorname{diag}\left(b_{1}(\omega), \ldots, b_{m}(\omega)\right) \in \Re^{m \times m}$ are diagonal matrices whose ith diagonal elements are given by

$$
a_{i}(\omega)=\left\{\begin{array}{c}
1, \text { if }-g_{i}(x)<\lambda_{i}, \\
0, \text { if }-g_{i}(x)>\lambda_{i}, \\
\mu_{i}, \text { if }-g_{i}(x)=\lambda_{i},
\end{array} \text { and } b_{i}(\omega)=\left\{\begin{array}{cl}
0, & \text { if }-g_{i}(x)<\lambda_{i} \\
1, & \text { if }-g_{i}(x)>\lambda_{i} \\
1-\mu_{i}, & \text { if }-g_{i}(x)=\lambda_{i}
\end{array}\right.\right.
$$

for any $\mu_{i} \in[0,1]$.

Proof. The first $n$ components of the vector function $\Phi$ are continuously differentiable, so the expression for the first $n$ columns of $H$ readily follows. Then, consider the last $m$ columns. Use the fact that

$$
\partial \phi(-g(x), \lambda)^{T} \subset \partial \varphi\left(-g_{1}(x), \lambda_{1}\right)^{T} \times \cdots \times \partial \varphi\left(-g_{m}(x), \lambda_{m}\right)^{T},
$$

if $i$ is such that $-g_{i}(x) \neq \lambda_{i}$, then $\phi$ is continuously differentiable at $\left(-g_{i}(x), \lambda_{i}\right)$ and the expression for the $(n+i)$ th column of $H$ follows. If instead $-g_{i}(x)=\lambda_{i}$, then, using the definition of the B-subdifferential, it follows that

$$
\partial_{B} \varphi\left(-g_{i}(x), \lambda_{i}\right)^{T}=\left\{\left(-\nabla g_{i}(x)^{T}, 0\right),\left(0, e_{i}^{T}\right)\right\} .
$$

Taking the convex hull, we get

$$
\partial \varphi\left(-g_{i}(x), \lambda_{i}\right)^{T}=\left\{\left(-\mu_{i} \nabla g_{i}(x)^{T},\left(1-\mu_{i}\right) e_{i}^{T}\right) \mid \mu_{i} \in[0,1]\right\} .
$$

This gives the representation of $H \in \partial \Phi(\omega)^{T}$. 
Our next aim is to establish conditions guaranteeing that all elements in the generalized Jacobian $\partial \Phi(\omega)$ at a point $\omega=(x, \lambda)$ satisfying $\Phi(\omega)=0$ are nonsingular.

Theorem 3.1 Let $\omega^{*}=\left(x^{*}, \lambda^{*}\right) \in \Re^{n+m}$ be a solution of the system $\Phi(\omega)=0$. Consider the following two statements:

(a) The strong second-order sufficient condition and the linear independence constraint qualification (LICQ) for VI $(F, X)$ holds at $x^{*}$.

(b) Any element in $\partial \Phi\left(\omega^{*}\right)$ is nonsingular.

It holds that (a) $\Rightarrow$ (b).

Proof. For the sake of notational simplicity, let us define the following subsets of the index set $I:=\{1, \ldots, m\}$,

$$
I_{0}:=\left\{i \mid g_{i}\left(x^{*}\right)=0, \lambda_{i}^{*} \geq 0\right\}, \quad I_{<}:=\left\{i \mid g_{i}\left(x^{*}\right)<0, \lambda_{i}^{*}=0\right\} .
$$

Moreover, we need

$$
\begin{array}{ll}
I_{00}:=\left\{i \mid g_{i}\left(x^{*}\right)=0, \lambda_{i}^{*}=0\right\}, & I_{+}:=\left\{i \mid g_{i}\left(x^{*}\right)=0, \lambda_{i}^{*}>0\right\}, \\
I_{01}:=\left\{i \in I_{00} \mid \mu_{i}=1\right\}, & I_{02}:=\left\{i \in I_{00} \mid \mu_{i} \in(0,1)\right\}, \\
I_{03}:=\left\{i \in I_{00} \mid \mu_{i}=0\right\} . &
\end{array}
$$

The following relationships between these index sets can easily be seen to hold:

$$
I=I_{0} \cup I_{<,} \quad I_{0}=I_{00} \cup I_{+}, \quad I_{00}=I_{01} \cup I_{02} \cup I_{03} .
$$

Using a suitable reordering of the constraints, every element $H \in \partial \Phi\left(\omega^{*}\right)^{T}$ has the following structure:

$$
H=\left[\begin{array}{cccccc}
\nabla_{x} L\left(\omega^{*}\right) & -\nabla g_{+}\left(x^{*}\right) & -\nabla g_{01}\left(x^{*}\right)-\nabla g_{02}\left(x^{*}\right) D_{a}\left(\omega^{*}\right)_{02} & 0 & 0 \\
\nabla g_{+}\left(x^{*}\right)^{T} & 0 & 0 & 0 & 0 & 0 \\
\nabla g_{01}\left(x^{*}\right)^{T} & 0 & 0 & 0 & 0 & 0 \\
\nabla g_{02}\left(x^{*}\right)^{T} & 0 & 0 & D_{b}\left(\omega^{*}\right)_{02} & 0 & 0 \\
\nabla g_{03}\left(x^{*}\right)^{T} & 0 & 0 & 0 & I & 0 \\
\nabla g_{<}\left(x^{*}\right)^{T} & 0 & 0 & 0 & 0 & I
\end{array}\right],
$$

where $D_{a}\left(\omega^{*}\right)_{02}$ and $D_{b}\left(\omega^{*}\right)_{02}$ are positive definite diagonal matrices. Note that we abbreviated $g_{I_{+}}$etc. by $g_{+}$etc. in (3.5). It is obvious that $H$ is nonsingular if and only if the following matrix is nonsingular,

$$
\left[\begin{array}{cccccc}
\nabla_{x} L\left(\omega^{*}\right) & -\nabla g_{+}\left(x^{*}\right) & -\nabla g_{01}\left(x^{*}\right) & -\nabla g_{02}\left(x^{*}\right) & 0 & 0 \\
\nabla g_{+}\left(x^{*}\right)^{T} & 0 & 0 & 0 & 0 & 0 \\
\nabla g_{01}\left(x^{*}\right)^{T} & 0 & 0 & 0 & 0 & 0 \\
\nabla g_{02}\left(x^{*}\right)^{T} & 0 & 0 & D_{b}\left(\omega^{*}\right)_{02} D_{a}\left(\omega^{*}\right)_{02}^{-1} & 0 & 0 \\
\nabla g_{03}\left(x^{*}\right)^{T} & 0 & 0 & 0 & I & 0 \\
\nabla g_{<}\left(x^{*}\right)^{T} & 0 & 0 & 0 & 0 & I
\end{array}\right] .
$$

In turn, this matrix is nonsingular if and only if the following matrix is nonsingular:

$$
\left[\begin{array}{cccc}
\nabla_{x} L\left(\omega^{*}\right) & -\nabla g_{+}\left(x^{*}\right) & -\nabla g_{01}\left(x^{*}\right) & -\nabla g_{02}\left(x^{*}\right) \\
\nabla g_{+}\left(x^{*}\right)^{T} & 0 & 0 & 0 \\
\nabla g_{01}\left(x^{*}\right)^{T} & 0 & 0 & 0 \\
\nabla g_{02}\left(x^{*}\right)^{T} & 0 & 0 & D_{b}\left(\omega^{*}\right)_{02} D_{a}\left(\omega^{*}\right)_{02}^{-1}
\end{array}\right] .
$$


Let $\left(\Delta x_{1}, \Delta x_{2}, \Delta x_{3}, \Delta x_{4}\right) \in \mathfrak{R}^{n} \times \mathfrak{R}^{\left|I_{+}\right|} \times \mathfrak{R}^{\left|I_{01}\right|} \times \mathfrak{R}^{\left|I_{02}\right|}$ be such that

$$
\left[\begin{array}{cccc}
\nabla_{x} L\left(\omega^{*}\right) & -\nabla g_{+}\left(x^{*}\right) & -\nabla g_{01}\left(x^{*}\right) & -\nabla g_{02}\left(x^{*}\right) \\
\nabla g_{+}\left(x^{*}\right)^{T} & 0 & 0 & 0 \\
\nabla g_{01}\left(x^{*}\right)^{T} & 0 & 0 & 0 \\
\nabla g_{02}\left(x^{*}\right)^{T} & 0 & 0 & D_{b}\left(\omega^{*}\right)_{02} D_{a}\left(\omega^{*}\right)_{02}^{-1}
\end{array}\right]\left[\begin{array}{c}
\Delta x_{1} \\
\Delta x_{2} \\
\Delta x_{3} \\
\Delta x_{4}
\end{array}\right]=0,
$$

we know that

$$
\begin{gathered}
\nabla_{x} L\left(\omega^{*}\right) \Delta x_{1}-\nabla g_{+}\left(x^{*}\right) \Delta x_{2}-\nabla g_{01}\left(x^{*}\right) \Delta x_{3}-\nabla g_{02}\left(x^{*}\right) \Delta x_{4}=0, \\
\nabla g_{+}\left(x^{*}\right)^{T} \Delta x_{1}=0, \\
\nabla g_{01}\left(x^{*}\right)^{T} \Delta x_{1}=0, \\
\nabla g_{02}\left(x^{*}\right)^{T} \Delta x_{1}+\left[D_{b}\left(\omega^{*}\right)_{02} D_{a}\left(\omega^{*}\right)_{02}^{-1}\right] \Delta x_{4}=0 .
\end{gathered}
$$

By the first, second and third equations of (3.8), we obtain that

$$
\begin{aligned}
0= & \left\langle\Delta x_{1}, \nabla_{x} L\left(\omega^{*}\right) \Delta x_{1}-\nabla g_{+}\left(x^{*}\right) \Delta x_{2}-\nabla g_{01}\left(x^{*}\right) \Delta x_{3}-\nabla g_{02}\left(x^{*}\right) \Delta x_{4}\right\rangle \\
= & \left\langle\Delta x_{1}, \nabla_{x} L\left(\omega^{*}\right) \Delta x_{1}\right\rangle-\left\langle\Delta x_{1}, \nabla g_{+}\left(x^{*}\right) \Delta x_{2}\right\rangle-\left\langle\Delta x_{1}, \nabla g_{01}\left(x^{*}\right) \Delta x_{3}\right\rangle \\
& -\left\langle\Delta x_{1}, \nabla g_{02}\left(x^{*}\right) \Delta x_{4}\right\rangle \\
= & \left\langle\Delta x_{1}, \nabla_{x} L\left(\omega^{*}\right) \Delta x_{1}\right\rangle-\left\langle\Delta x_{1}, \nabla g_{02}\left(x^{*}\right) \Delta x_{4}\right\rangle,
\end{aligned}
$$

which, together with the last equation of (3.8), implies that

$$
\left\langle\Delta x_{1}, \nabla_{x} L\left(\omega^{*}\right) \Delta x_{1}\right\rangle=-\Delta x_{4}^{T}\left[D_{b}\left(\omega^{*}\right)_{02} D_{a}\left(\omega^{*}\right)_{02}^{-1}\right] \Delta x_{4} \leq 0 .
$$

From the second equation of (3.8), we know that

$$
\Delta x_{1} \in \operatorname{aff}\left(C\left(x^{*}\right)\right),
$$

where $C\left(x^{*}\right)$ denotes the critical cone of $\operatorname{VI}(F, X)$. Then, by (3.9) and the strong second-order sufficient condition that

$$
\Delta x_{1}=0 .
$$

Thus, the first equation of (3.8) reduces to

$$
\nabla g_{+}\left(x^{*}\right) \Delta x_{2}+\nabla g_{01}\left(x^{*}\right) \Delta x_{3}+\nabla g_{02}\left(x^{*}\right) \Delta x_{4}=0 .
$$

By the LICQ for $\operatorname{VI}(F, X)$, we have

$$
\Delta x_{2}=0, \Delta x_{3}=0, \text { and } \Delta x_{4}=0 .
$$

This together with $\Delta x_{1}=0$ shows that the matrix (3.6) is nonsingular, and then, $H$ is nonsingular.

Now, we are able to apply Theorem 3.1 to some classes of GNEPs.

Proposition 3.1 Let $\omega^{*}=\left(x^{*}, \lambda^{*}\right) \in \Re^{n+m}$ satisfying $\Phi\left(\omega^{*}\right)=0$, for all $v=1, \ldots ., N$ the payoff functions $\theta^{v}$ are separable, that is

$$
\theta^{v}(x)=f^{v}\left(x^{\nu}\right)+h^{v}\left(x^{-v}\right)
$$

where $f^{v}: \Re^{n_{v}} \rightarrow \Re$ is stongly convex and $h^{v}: \Re^{n-n_{v}} \rightarrow \Re$. Assume that LICQ holds at $x^{*}$. Then all elements $H \in \partial \Phi\left(\omega^{*}\right)$ are nonsingular. 
Proof. We know that

$$
F\left(x^{*}\right)=\left(\begin{array}{c}
\nabla_{x^{1}} \theta^{1}\left(x^{*}\right) \\
\vdots \\
\nabla_{x^{N}} \theta^{N}\left(x^{*}\right)
\end{array}\right),
$$

then, by the definition of $\theta^{v}(\cdot)$, we have

$$
\begin{array}{r}
\nabla F(x *)=\left(\begin{array}{cccc}
\nabla_{x^{1} x^{1}}^{2} \theta^{1}(x *) & \nabla_{x^{1} x^{2}}^{2} \theta^{1}(x *) & \cdots & \nabla_{x^{1} x^{N}}^{2} \theta^{1}(x *) \\
\nabla_{x^{2} x^{1}}^{2} \theta^{2}(x *) & \nabla_{x^{2} x^{2}}^{2} \theta^{2}(x *) & \cdots & \nabla_{x^{2} x^{N}}^{2} \theta^{2}(x *) \\
\vdots & \vdots & \ddots & \\
\nabla_{x^{N} x^{1}}^{2} \theta^{N}(x *) & \nabla_{x^{N} x^{2}}^{2} \theta^{N}(x *) & \cdots & \nabla_{x^{N} x^{N}}^{2} \theta^{N}(x *)
\end{array}\right) \\
=\left(\begin{array}{cccc}
\nabla_{x^{1} x^{1}}^{2} f^{1}\left(x^{*, 1}\right) & & \\
& \nabla_{x^{2} x^{2}}^{2} f^{2}\left(x^{*, 2}\right) & & \\
& & \ddots & \\
& & \nabla_{x^{N} x^{N}}^{2} f^{N}\left(x^{*, N}\right)
\end{array}\right)
\end{array}
$$

By the strong convexity of $f^{\prime}$, we can conclude that $\nabla F\left(x^{*}\right)$ is positive definite.

From $\lambda_{i}^{*} \geq 0$ and the convexity of $g_{i}$, we obtain that

$$
\nabla_{x}\left(\nabla g\left(x^{*}\right) \lambda^{*}\right)=\sum_{i=1}^{m} \lambda_{i}^{*} \nabla^{2} g_{i}\left(x^{*}\right)
$$

is positive semidefinite, which together with $\nabla F\left(x^{*}\right)$ is positive definite implies that

$$
\nabla_{x} L\left(\omega^{*}\right)=\nabla F\left(x^{*}\right)+\sum_{i=1}^{m} \lambda_{i}^{*} \nabla^{2} g_{i}\left(x^{*}\right)
$$

is positive definite. Thus, the strong second-order sufficient condition for the $\operatorname{VI}(F$, $X)$ holds at $x^{*}$. From Theorem 3.1, we obtain any element in $\partial \Phi\left(\omega^{*}\right)$ is nonsingular.

Proposition 3.2 Let $\omega^{*}=\left(x^{*}, \lambda^{*}\right) \in \Re^{n+m}$ be such that $\Phi\left(\omega^{*}\right)=0$. Consider the case where the payoff functions are quadratic, i.e. for all $v=1, \ldots, N$ one has

$$
\theta^{\nu}(x):=\frac{1}{2}\left(x^{\nu}\right)^{T} A_{\nu v} x^{\nu}+\sum_{\mu=1, \mu \neq \nu}^{N}\left(x^{\nu}\right)^{T} A_{\nu \mu} x^{\mu},
$$

where the matrices $A_{v \mu} \in \Re^{n_{v}} \times \Re^{n_{\mu}}$ and $A_{v v}$ are symmetric. Suppose that LICQ holds at $x^{*}$, and

$$
\mathbf{B}:=\left[\begin{array}{cccc}
A_{11} & A_{12} & \cdots & A_{1 N} \\
A_{21} & A_{22} & \cdots & A_{2 N} \\
\vdots & \vdots & \ddots & \vdots \\
A_{N 1} & A_{N 2} & \cdots & A_{N N}
\end{array}\right]
$$

is positive definite. Then all the elements in the generalized Jacobian $\partial \Phi\left(\omega^{*}\right)$ are nonsingular.

Proof. We show that $\nabla_{x} L\left(\omega^{*}\right)$ is positive definite, which implies that the strong second order sufficient condition for the $\operatorname{VI}(F, X)$ holds at $x^{*}$, and then apply Theorem 3.1. To this end, first note that 


$$
F\left(x^{*}\right)=\left(\begin{array}{c}
\nabla_{x^{1}} \theta^{1}\left(x^{*}\right) \\
\nabla_{x^{2}} \theta^{2}\left(x^{*}\right) \\
\vdots \\
\nabla_{x^{N}} \theta^{N}\left(x^{*}\right)
\end{array}\right)=\left(\begin{array}{c}
\sum_{\mu=1}^{N} A_{1 \mu} x^{\mu} \\
\sum_{\mu=1}^{N} A_{2 \mu} x^{\mu} \\
\vdots \\
\sum_{\mu=1}^{N} A_{N \mu} x^{\mu}
\end{array}\right) .
$$

Moreover,

$$
\nabla F\left(x^{*}\right)=\left(\begin{array}{cccc}
A_{11} & A_{12} & \cdots & A_{1 N} \\
A_{21} & A_{22} & \cdots & A_{21} \\
\cdots & & \\
A_{N 1} & A_{N 1} & \cdots & A_{N N}
\end{array}\right),
$$

which together with $\lambda_{i}^{*} \geq 0$ and the convexity of $g_{i}$ implies that

$$
\nabla_{x}\left(\nabla g\left(x^{*}\right) \lambda^{*}\right)=\sum_{i=1}^{m} \lambda_{i}^{*} \nabla^{2} g_{i}\left(x^{*}\right)
$$

is positive semidefinite. Hence, we obtain that $\nabla_{x} L\left(\omega^{*}\right)$ is positive definite. The statement therefore follows from Theorem 3.1.

\section{Numerical illustrations}

Here, we want to illustrate the performance of the VI method on some GNEPs taken from the literature. To this end, we use a nonsmooth Newton method to the nonlinear system of equations $\Phi(\omega)=0$. The globalization strategy is based on the merit function

$$
\Psi(\omega):=\frac{1}{2} \Phi(\omega)^{T} \Phi(\omega)
$$

A simple Armijo-type line search is used in the algorithm and we switch to the steepest direction whenever the generalized Newton direction is not computable or does not satisfy a sufficient decrease condition.

\section{Algorithm 4.1}

Step 0 Choose $\omega^{0}=\left(x^{0}, \lambda^{0}\right) \in \Re^{n+m}, \rho>0, \kappa>2, \sigma \in\left(0, \frac{1}{2}\right), \beta \in(0,1), \varepsilon \geq 0$, and set $k=0$.

Step 1 If $\left\|\nabla \Psi\left(\omega^{k}\right)\right\| \leq \varepsilon$, stop.

Step 2 Select an element $H_{k} \in \partial_{B} \Phi\left(\omega^{k}\right)$. Find a solution $d^{k}$ of the linear system

$$
H_{k} d=-\Phi\left(\omega^{k}\right)
$$

If system (4.1) is not solvable or if $d^{k}$ does not satisfy the condition

$$
\nabla \Psi\left(\omega^{k}\right)^{T} d^{k} \leq-\rho\left\|d^{k}\right\|^{\kappa}
$$

then set

$$
d^{k}=-\nabla \Psi\left(\omega^{k}\right)
$$

Step 3 Let $t^{k}$ be the greatest number in $\left\{\beta^{j} \mid j=0,1,2, \ldots\right\}$ such that

$$
\Psi\left(\omega^{k}+t^{k} d^{k}\right) \leq \Psi\left(\omega^{k}\right)+t^{k} \sigma \nabla \Psi\left(\omega^{k}\right)^{T} d^{k}
$$


Step 4 Set $\omega^{k+1}=\omega^{k}+t^{k} d^{k}, k=k+1$ and go to step 1 .

The following result about the convergence property of Algorithm 4.1 comes from [18] directly.

Theorem 4.1 Assume that Algorithm 4.1 does not terminate within a finite number of iterations, let $\left\{\omega^{k}\right\}$ be generated by Algorithm 4.1 having an accumulation point $\omega^{*}$, then $\omega^{*}$ is a stationary point of $\Psi$. Moreover, if $\omega^{*}$ is a BD-regular solution of the system $\Phi(\omega)=0$, then $\left\{\omega^{k}\right\}$ convergence to $\omega^{*} Q$-superlinearly.

We applied MATLAB 7.0 to some problems of GNEPs. The method is terminated whenever $\left\|\nabla \Psi\left(\omega^{k}\right)\right\|<\varepsilon$ with $\varepsilon:=10^{-7}$. The computational results are summarized in Tables 1, 2, and 3, which indicate that the proposed method produces good approximate solutions.

Example 4.1 This test problem is the internet switching model introduced by Facchinei et al. [19]. The payoff function of each user is given by

$$
\theta^{v}(x):=\frac{x^{v}}{B}-\frac{x^{v}}{\sum_{v=1}^{N} x^{\nu}}
$$

with constraints $x^{v} \geq 0.01, v=1, \ldots, N$ and $\sum_{v=1}^{N} x^{v} \leq B$. According to [20], we also set $N=10, B=1$ and use the starting point $x^{0}=(0.1,0.1,0.1, \ldots,)^{T} \in \mathfrak{R}^{10}$. The exact solution of this problem is $x^{*}=(0.09,0.09, \ldots, 0.09)^{T}$. We only state the first three components of the iteration vectors in Table 1.

Example 4.2 This example is the river basin pollution game taken from [5] and is also analyzed by Heusinger and Kanzow [20]. There are three players, each controlling a single variable $x^{\nu} \in \Re$. The objective functions are

$$
\theta^{v}(x):=x^{v}\left(c_{1 v}+c_{2 v} x^{v}-d_{1}+d_{2}\left(x^{1}+x^{2}+x^{3}\right)\right)
$$

for $v=1,2,3$, and the constraints are

$$
\begin{aligned}
& \mu_{11} e_{1} x^{1}+\mu_{21} e_{2} x^{2}+\mu_{31} e_{3} x^{3} \leq K 1, \\
& \mu_{12} e_{1} x^{1}+\mu_{22} e_{2} x^{2}+\mu_{32} e_{3} x^{3} \leq K 2 .
\end{aligned}
$$

The economic constants $d_{1}$ and $d_{2}$ determine the inverse demand law and set to 3.0 and 0.01, respectively. Values for constants $c_{1, v}, c_{2, v}, e_{v}, \mu_{v, 1}$ and $\mu_{V, 2}$ are given in the following table, and $K_{1}=K_{2}=100$. Table 2 for the corresponding numerical results.

Table 1 Numerical results for Example 4.1

\begin{tabular}{lllll}
\hline $\boldsymbol{k}$ & $x_{1}^{k}$ & $x_{2}^{k}$ & $x_{3}^{k}$ & Stepsize \\
\hline 0 & 0.100000 & 0.100000 & 0.100000 & 0 \\
1 & 0.086298 & 0.086298 & 0.086298 & 0.015224 \\
2 & 0.095471 & 0.095471 & 0.095471 & 0.015224 \\
3 & 0.087856 & 0.087856 & 0.087856 & 0.015224 \\
4 & 0.093390 & 0.093390 & 0.093390 & 0.015224 \\
5 & 0.088716 & 0.088716 & 0.088716 & 0.015224 \\
$\vdots$ & $\vdots$ & $\vdots$ & $\vdots$ & $\vdots$ \\
10 & 0.091365 & 0.091365 & 0.091365 & 0.015224 \\
$\vdots$ & $\vdots$ & $\vdots$ & $\vdots$ & $\vdots$ \\
20 & 0.089952 & 0.089952 & 0.089952 & 0.302500 \\
21 & 0.089968 & 0.089968 & 0.089968 & 0.008373 \\
\hline
\end{tabular}


Table 2 Numerical results for Example 4.2

\begin{tabular}{lllll}
\hline $\boldsymbol{k}$ & $\boldsymbol{x}_{1}^{k}$ & $\boldsymbol{x}_{2}^{k}$ & $\boldsymbol{x}_{3}^{k}$ & Stepsize \\
\hline 0 & 0.000000 & 0.000000 & 0.000000 & 0.0000 \\
1 & 9.208951 & 2.481282 & 8.931660 & 0.166375 \\
2 & 11.531188 & 3.106991 & 11.183971 & 0.050328 \\
3 & 12.744136 & 3.433811 & 12.360396 & 0.027680 \\
4 & 13.100896 & 3.529937 & 12.706414 & 0.008373 \\
5 & 13.159756 & 3.545796 & 12.763501 & 0.001393 \\
6 & 13.177536 & 3.550587 & 12.780746 & 0.000421 \\
7 & 13.182912 & 3.552036 & 12.785960 & 0.000127 \\
8 & 21.175468 & 16.026854 & 2.771656 & 1 \\
9 & 21.149274 & 16.027708 & 2.732634 & 1 \\
10 & 21.144948 & 16.027849 & 2.726189 & 1 \\
11 & 21.144796 & 16.027853 & 2.725963 & 1 \\
\hline
\end{tabular}

Example 4.3 We use Algorithm 4.1 to solve a class of problems in which for each player $v$, his payoff function $\theta^{v}(\cdot)$ is quadratic, that is

$$
\theta^{\nu}(x):=\frac{1}{2}\left(x^{\nu}\right)^{T} A_{\nu v} x^{\nu}+\sum_{\mu=1, \mu \neq \nu}^{N}\left(x^{\nu}\right)^{T} A_{\nu \mu} x^{\mu}
$$

for certain matrices $A_{v \mu} \in \mathbf{R}^{n_{v}} \times \mathbf{R}^{n_{\mu}}$ such that the diagonal block $A_{v v}$ are symmetric. Let

$$
\mathbf{B}:=\left[\begin{array}{cccc}
A_{11} & A_{12} & \cdots & A_{1 N} \\
A_{21} & A_{22} & \cdots & A_{2 N} \\
\vdots & \vdots & \ddots & \vdots \\
A_{N 1} & A_{N 2} & \cdots & A_{N N}
\end{array}\right]
$$

be positive definite, The strategy space $X$ is defined by some linear constraints. For convenience, we set the elements of $x^{0}$ all 1 . The elements of $\lambda^{0}$ all 0 . We set other parameters in the algorithm as $\rho=10^{-8}, \kappa=2.1, \sigma=10^{-4}, \beta=0.55$. Our numerical results are reported in Table 3, where Iter., Func, Res0. and Res*. stand for, respectively, the number of iterations, the number of function evaluations, the residual $\|\nabla \Psi(\cdot)\|$ at the starting point and the residual $\|\nabla \Psi(\cdot)\|$ at the final iterate of implementation.

Table 3 Numerical results for Example 4.3

\begin{tabular}{llllll}
\hline Dim & CPU time $(\mathbf{s})$ & Iter. & Func. & Res0. & Res*. \\
\hline 10 & 0.0470 & 6 & 15 & $5.227683 \mathrm{e}+004$ & $1.028557 \mathrm{e}-009$ \\
50 & 0.0520 & 7 & 20 & $1.529682 \mathrm{e}+008$ & $1.752247 \mathrm{e}-010$ \\
100 & 0.3250 & 7 & 24 & $5.052647 \mathrm{e}+009$ & $6.559316 \mathrm{e}-013$ \\
300 & 0.6340 & 9 & 33 & $1.230922 \mathrm{e}+012$ & $2.016572 \mathrm{e}-010$ \\
500 & 2.6720 & 11 & 43 & $1.581871 \mathrm{e}+013$ & $1.446427 \mathrm{e}-012$ \\
1000 & 13.2189 & 9 & 38 & $5.002778 \mathrm{e}+014$ & $1.458512 \mathrm{e}-009$ \\
1500 & 39.4220 & 9 & 34 & $3.800590 \mathrm{e}+015$ & $3.468333 \mathrm{e}-013$ \\
2000 & $1.0953 \mathrm{e}+002$ & 11 & 50 & $1.601577 \mathrm{e}+016$ & $2.619540 \mathrm{e}-014$ \\
2500 & $2.2250 \mathrm{e}+002$ & 12 & 50 & $4.889072 \mathrm{e}+016$ & $3.492963 \mathrm{e}-014$ \\
3000 & $3.4442 \mathrm{e}+002$ & 11 & 47 & $1.216867 \mathrm{e}+017$ & $1.782483 \mathrm{e}-013$ \\
\hline
\end{tabular}




\begin{tabular}{llllll}
\hline (Continued) & \multicolumn{6}{l}{} \\
\hline playerv & $\boldsymbol{c}_{\mathbf{1 , \boldsymbol { v }}}$ & $\boldsymbol{c}_{\mathbf{2 , \boldsymbol { v }}}$ & $\boldsymbol{e}_{\boldsymbol{v}}$ & $\boldsymbol{\mu}_{\boldsymbol{v}, \mathbf{1}}$ & $\boldsymbol{\mu}_{\boldsymbol{v}, \mathbf{2}}$ \\
\hline 1 & 0.10 & 0.01 & 0.50 & 6.5 & 4.583 \\
2 & 0.12 & 0.05 & 0.25 & 5.0 & 6.250 \\
3 & 0.15 & 0.01 & 0.75 & 5.5 & 3.750 \\
\hline
\end{tabular}

The numerical experiments show that the method proposed in this article is implementable and effective.

\section{Acknowledgements}

The research was supported by the Fundamental Innovation Methods Funds under Project No. $2010 \mathrm{IM020300}$ and the Technology Research of Inner Mongolia under Project No. 20100915.

\section{Author details}

${ }^{1}$ Institute of ORCT, School of Mathematical Sciences, Dalian University of Technology, Dalian 116024, China

${ }^{2}$ Management College of Inner Mongolia University of Technology, Hohhot 010051, China

\section{Authors' contributions}

$\mathrm{JH}$ and Z-CW carried out the design of the study and performed the analysis. ZW participated in its design and coordination. All authors read and approved the final manuscript.

\section{Competing interests}

The authors declare that they have no competing interests.

Received: 12 October 2011 Accepted: 9 March 2012 Published: 9 March 2012

\section{References}

1. Debreu, G: A social equilibrium existence theorem. Proc Natl Acad Sci USA. 38, 886-893 (1952). doi:10.1073/ pnas.38.10.886

2. Altman, E, Wynter, L: Equilibrium, games, and pricing in transportation and telecommunication networks. Netw Spat Econ. 4, 7-21 (2004)

3. $\mathrm{Hu}, \mathrm{X}, \mathrm{Ralph}, \mathrm{D}$ : Using EPECs to model bilevel games in restructured electricity markets with locational prices. Oper Res 55, 809-827 (2007). doi:10.1287/opre.1070.0431

4. Krawczyk, JB: Coupled constraint Nash equilibria in enviromental games. Resour Energy Econ. 27, 157-181 (2005). doi:10.1016/j.reseneeco.2004.08.001

5. Krawczyk, JB, Uryasev, S: Relaxation algorithms to find Nash equilibria with economic applications. Environ Model Assess. 5, 63-73 (2000). doi:10.1023/A:1019097208499

6. Uryasev, S, Rubinstein, RY: On relaxation algorithms in computation of noncoopera-tive equilibria. IEEE Trans Autom Control. 39, 1263-1267 (1994), doi:10.1109/9.293193

7. Flam, SD, Ruszczynski, A: Noncooperative convex games: computing equilibrium by partial regulalization. IIASA Working Paper Austria. 94-142 (1994)

8. Gürkan, G, Pang, JS: Approximations of Nash equilibria. Math Program. 117, 223-253 (2009). doi:10.1007/s10107-0070156-y

9. Heusinger, AV, Kanzow, C: Optimization reformulations of the generalized Nash equilibrium problem using NikaidoIsoda-type functions. Comput Optim Appl. 43, 353-377 (2009). doi:10.1007/s10589-007-9145-6

10. Facchinei, F, Pang, JS: Finite-dimensional variational inequalities and complementarity problems. I, Springer, New York (2003)

11. Facchinei, F, Pang, JS: Finite-dimensional variational inequalities and complementarity problems. Springer, New Yorkll (2003)

12. Harker, PT: Generalized Nash games and quasi-variational inequalities. Eur J Oper Res. 54, $81-94$ (1991). doi:10.1016/ 0377-2217(91)90325-P

13. Facchinei, F, Fisher, A, Piccialli, V: On generalized Nash games and variational inequalities. Oper Res Lett. 35, 159-164 (2007). doi:10.1016/j.orl.2006.03.004

14. Qi, L: Convergence analysis of some algorithms for solving nonsmooth equations. Math Oper Res. 18, $227-244$ (1993). doi:10.1287/moor.18.1.227

15. Clarke, FH: Optimization and Nonsmooth Analysis. John Wiley, New York (1983)

16. Mifflin, R: Semismooth and semiconvex functions in constrained optimization. SIAM J Control Optim. 15, 959-972 (1977). doi:10.1137/0315061

17. Qi, L, Sun, J: A nonsmooth version of Newton's method. Math Program. 58, 353-368 (1993). doi:10.1007/BF01581275

18. Luca, TD, Facchinei, F, Kanzow, C: A semismooth equation approach to the solution of nonlinear complementarity problems. Math Program. 75, 407-439 (1996)

19. Facchinei, F, Fisher, A, Piccialli, V: Generalized Nash equilibrium problems and Newton methods. Math Program. 117, 163-194 (2009). doi:10.1007/s10107-007-0160-2 
20. Heusinger, AV, Kanzow, C: Relaxation methods for generalized Nash equilibrium problems with inexact line search. J Optim Theory Appl. 143, 159-183 (2009). doi:10.1007/s10957-009-9553-0

doi:10.1186/1029-242X-2012-60

Cite this article as: Hou et al:: A variational inequality method for computing a normalized equilibrium in the generalized Nash game. Journal of Inequalities and Applications 2012 2012:60.

Submit your manuscript to a SpringerOpen ${ }^{\odot}$ journal and benefit from:

- Convenient online submission

- Rigorous peer review

- Immediate publication on acceptance

- Open access: articles freely available online

- High visibility within the field

- Retaining the copyright to your article

Submit your next manuscript at $\gg$ springeropen.com 